
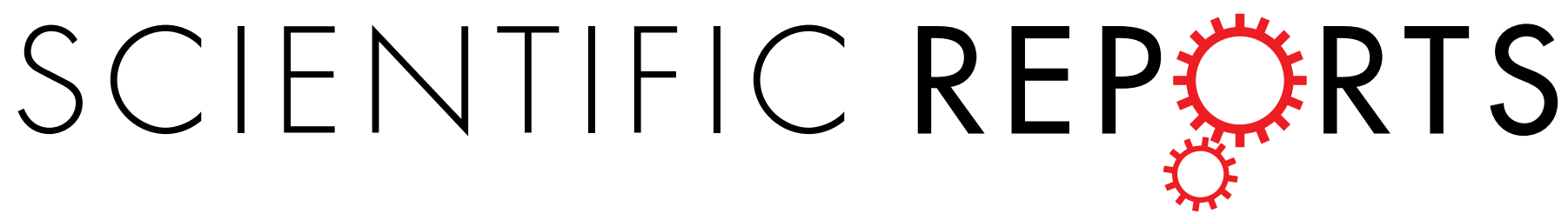

\title{
OPEN Genetic polymorphisms in MTR are associated with non-syndromic congenital heart disease from a \\ family-based case-control study in the Chinese population
}

Received: 22 November 2018

Accepted: 11 March 2019

Published online: 25 March 2019
Changfei Deng ${ }^{1,2}$, Ying Deng ${ }^{1,2}$, Liang Xie ${ }^{2,3,4}$, LiYu ${ }^{5}$, Lijun Liu ${ }^{2}$, Hanmin Liu ${ }^{2,3,4} \&$ Li Dai $^{1,2}$

Genetic polymorphisms of folate pathway genes have been reported to be associated with congenital heart diseases (CHDs); however, the results remain conflicting. We conducted a family-based casecontrol study, which included $160 \mathrm{CHD}$ case-parent triads and 208 control-parent triads to explore the association of 18 genetic variants of seven folate metabolism-related genes with the risk of CHDs. The $M T R$ C allele of rs $1770449(\mathrm{OR}=1.961,95 \% \mathrm{Cl}: 1.379-2.788)$ and the $M T R$ A allele of rs 1050993 $(\mathrm{OR}=1.994,95 \% \mathrm{Cl}: 1.401-2.839)$ in infants were associated with an increased risk of CHDs. Overtransmission of SNPs rs1770449 and rs1050993 and haplotype CAA (rs1770449-rs1805087-rs1050993) in MTR were detected in total CHDs. The above mentioned associations of MTR with CHDs were also observed in septal defects and conotruncal heart defects subgroups. Without maternal periconceptional folate intake, the risk of CHDs among women carrying the rs1770449 "CT or CC" genotype or the rs1050993 "AG or AA" genotype in MTR was 3.262(95\%Cl: 1.656-6.429) or 3.263(95\%Cl: 1.656-6.429) times greater than the aOR in women carrying wild genotype, respectively. Our study suggests that MTR polymorphisms (rs1770449 and rs1050993) may be associated with the risk of CHDs and modify the relation between maternal folate intake and CHDs.

Congenital heart diseases (CHDs) represent the most common birth defect and the leading cause of infant deaths worldwide, affecting 0.6-9.1 per 1000 live births with substantial geographic variations ${ }^{1}$. In China, the prevalence of CHD is 8.98 per 1000 live births ${ }^{2}$ and approximately 1 to 4 of every 1000 births with an upward trend during $2000-2011^{3}$. The aetiology of non-syndromic CHD remains unknown. Many studies over the past decades suggested that the interaction of genetic and environmental factors contributed to the increasing risk of CHDs ${ }^{4-6}$, however, the results are still inconsistent ${ }^{7}$.

It is generally accepted that maternal periconceptional supplementation with folic acid could contribute to a $40-60 \%$ reduction in $\mathrm{CHDs}^{8-10}$. Furthermore, the results of some association studies on SNPs in the folate metabolism pathway have shown that several genetic variants in MTHFR, MTR and MTRR might be associated with $\mathrm{CHDs}^{11,12}$. However, several other studies reported contrasting results due to differences in study population, design method, etc. These results indicate that more studies are needed to elucidate the mechanism of genetic factors and maternal folic acid intake in $\mathrm{CHDs}^{7,13}$.

In the present study, we hypothesized that the perturbations of folate pathway genes are associated with a susceptibility to CHDs. A family-based case-control study was performed to illustrate (1) case-control association,

${ }^{1}$ National Center for Birth Defects Monitoring, West China Second University Hospital, Sichuan University, Chengdu, Sichuan, China. ${ }^{2}$ Key Laboratory of Birth Defects and Related Diseases of Women and Children (Sichuan University), Ministry of Education, Chengdu, Sichuan, China. ${ }^{3}$ Department of Pediatric Respiration, West China Second University Hospital, Sichuan University, Chengdu, Sichuan, China. ${ }^{4}$ The Vascular Remodeling and Developmental Defects Research Unit, West China Institute of Women and Children's Health, West China Second University Hospital, Sichuan University, Chengdu, Sichuan, China. ${ }^{5}$ Department of Pediatric Cardiology, West China Second University Hospital, Sichuan University, Chengdu, Sichuan, China. Changfei Deng and Ying Deng contributed equally. Correspondence and requests for materials should be addressed to L.D. (email: daili@scu.edu.cn) 


\begin{tabular}{|c|c|c|c|c|c|c|}
\hline \multirow[b]{2}{*}{ Characteristics } & \multicolumn{2}{|c|}{ Cases } & \multicolumn{2}{|c|}{ Controls } & \multirow[b]{2}{*}{$\chi^{2}$} & \multirow[b]{2}{*}{$P$} \\
\hline & No. & $\%$ & No. & $\%$ & & \\
\hline Maternal age(yrs) & & & & & 21.391 & $<0.001$ \\
\hline$<25$ & 33 & 20.63 & 13 & 6.25 & & \\
\hline $25-29$ & 75 & 46.88 & 92 & 44.23 & & \\
\hline $30-34$ & 41 & 25.63 & 79 & 37.98 & & \\
\hline$\geq 35$ & 11 & 6.88 & 24 & 11.54 & & \\
\hline \multicolumn{3}{|l|}{ Maternal education level } & & & 41.334 & $<0.001$ \\
\hline Junior school or less & 23 & 14.38 & 5 & 2.40 & & \\
\hline Senior high school & 30 & 18.75 & 12 & 5.77 & & \\
\hline College & 92 & 57.50 & 145 & 69.71 & & \\
\hline Master or advance & 15 & 9.38 & 46 & 22.12 & & \\
\hline \multicolumn{3}{|l|}{ Residence } & & & 43.531 & $<0.001$ \\
\hline Urban & 101 & 63.13 & 190 & 91.35 & & \\
\hline Rural & 59 & 36.88 & 18 & 8.65 & & \\
\hline \multicolumn{3}{|c|}{ Household income(rmb monthly) } & & & 46.794 & $<0.001$ \\
\hline$<3000$ & 26 & 16.25 & 4 & 1.92 & & \\
\hline 3000-5999 & 41 & 25.63 & 24 & 11.54 & & \\
\hline 6000-8999 & 46 & 28.75 & 104 & 50.00 & & \\
\hline 9000-11999 & 28 & 17.50 & 34 & 16.35 & & \\
\hline$\geq 12000$ & 19 & 11.88 & 42 & 20.19 & & \\
\hline Maternal folate intake & & & & & 8.736 & 0.003 \\
\hline Yes & 69 & 43.13 & 122 & 58.65 & & \\
\hline No & 91 & 56.88 & 86 & 41.35 & & \\
\hline Fetal gender & & & & & 0.004 & 0.949 \\
\hline Male & 81 & 50.63 & 106 & 50.96 & & \\
\hline Female & 79 & 49.38 & 102 & 49.04 & & \\
\hline
\end{tabular}

Table 1. Characteristics in the CHD case and control groups.

(2) transmission disequilibrium and (3) gene-environment interaction between genetic variants of seven key enzyme genes in the folate metabolic pathway and the risk of CHDs.

\section{Results}

Demographic characteristics. In our study, a total of 368 complete triads were analysed (160 CHD case-parent triads and 208 control-parent triads). Significant differences were found in case and control groups with respect to maternal age, maternal education level, residence, household income and maternal folate intake (Table 1).

Association between infant SNPs and CHDs. None of the genotype frequencies for 18 infant SNPs of seven folate pathway genes in the controls deviated from the Hardy-Weinberg equilibrium(Supplementary Table S1).

Table 2 shows the association between infant SNPs and the risk of CHDs. The C allele of rs1770449 and the A allele of rs 1050993 in MTR were associated with an increased risk of CHDs(aOR = 1.96, 95\%CI: 1.38-2.79; $\mathrm{aOR}=1.99,95 \% \mathrm{CI}: 1.40-2.84)$ after the false discovery rate (FDR) correction. Under additive model, the SNP rs 1770449 and $\mathrm{rs} 1050993(\mathrm{aOR}=1.87,95 \% \mathrm{CI}: 1.27-2.76$; $\mathrm{aOR}=1.90,95 \% \mathrm{CI}$ : $1.28-2.80)$ were associated with an increased risk of CHDs under FDR correction for multiple testing.

Transmission disequilibrium. Table 3 and Supplementary Table S2 present the transmission of genetic variants from heterozygous CHD case-parent triads. Over-transmission was observed in the SNPs rs1770449 and rs1050993 of MTR after the adjustment by the permutation test as follows: total CHDs (rs1770449: $\mathrm{OR}=2.28,95 \% \mathrm{CI}: 1.51-3.46$; rs 1050993: $\mathrm{OR}=2.28,95 \% \mathrm{CI}: 1.51-3.46)$, septal defects (SPD) (rs1770449: $\mathrm{OR}=2.32$, 95\%CI: $1.45-3.71$; rs 1050993: $\mathrm{OR}=2.32$, 95\%CI: $1.45-3.71)$, conotruncal heart defects (CTD) $(\mathrm{rs} 1770449: \mathrm{OR}=4.00,95 \% \mathrm{CI}: 2.07-7.75 ; \mathrm{rs} 1050993: \mathrm{OR}=4.00,95 \% \mathrm{CI}: 2.07-7.75)$.

Enhanced risk was found in haplotype CAA (rs1770449-rs1805087-rs1050993), while decreased risk was found in haplotype TAG in the total CHD, SPD and CTD groups (Table 4). Transmission of haplotypes in other related genes is shown in Supplementary Table S3.

Interaction between infant SNPs and maternal folate intake. Assuming a dominant genetic model, multiplicative-scale interactions between maternal folate intake and the SNPs rs1770449 $(B=-1.152$, Wals $=5.743)$ and $\mathrm{rs} 1050993(\mathrm{~B}=-1.116$, Wals $=5.386)$ in $M T R$ and the risk of CHDs were observed after adjusting for potential confounders. Without periconceptional maternal folate intake, the risk of CHDs among the women carrying rs1770449 "CT or CC" genotype or rs 1050993 "AG or AA" genotype in MTR was 3.262(95\%CI: 


\begin{tabular}{|c|c|c|c|c|c|c|c|}
\hline \multirow[b]{2}{*}{ Marker } & \multirow[b]{2}{*}{ Polymorphisms } & \multicolumn{2}{|c|}{ Cases } & \multicolumn{2}{|c|}{ Controls } & \multirow[b]{2}{*}{ aOR $(95 \% \mathrm{CI}) *$} & \multirow[b]{2}{*}{$P^{\$}$} \\
\hline & & No. & $\%$ & No. & $\%$ & & \\
\hline \multicolumn{8}{|l|}{ rs 1770449} \\
\hline & $\mathrm{T}$ & 228 & 71.3 & 345 & 82.9 & Ref. & \\
\hline & $\mathrm{C}$ & 92 & 28.8 & 71 & 17.1 & $1.961(1.379,2.788)$ & 0.003 \\
\hline \multirow{2}{*}{ Dominant } & TT & 82 & 51.3 & 141 & 67.8 & Ref. & \\
\hline & TC or CC & 78 & 48.8 & 67 & 32.2 & $1.835(1.159,2.907)$ & 0.130 \\
\hline \multirow{2}{*}{ Recessive } & TC or TT & 146 & 91.3 & 204 & 98.1 & Ref. & \\
\hline & $\mathrm{CC}$ & 14 & 8.8 & 4 & 1.9 & $4.759(1.452,15.600)$ & 0.190 \\
\hline Additive & - & - & - & - & - & $1.870(1.267,2.762)$ & 0.031 \\
\hline \multicolumn{8}{|l|}{ rs1050993 } \\
\hline & G & 228 & 71.3 & 346 & 83.2 & Ref. & \\
\hline & A & 92 & 28.8 & 70 & 16.8 & $1.994(1.401,2.839)$ & 0.003 \\
\hline \multirow{2}{*}{ Dominant } & GG & 82 & 51.3 & 142 & 68.3 & Ref. & \\
\hline & GA or AA & 78 & 48.8 & 66 & 31.7 & $1.873(1.182,2.969)$ & 0.130 \\
\hline \multirow{2}{*}{ Recessive } & GA or GG & 146 & 91.3 & 204 & 98.1 & Ref. & \\
\hline & AA & 14 & 8.8 & 4 & 1.9 & $4.759(1.452,15.600)$ & 0.190 \\
\hline Additive & - & - & - & - & - & $1.898(1.284,2.804)$ & 0.031 \\
\hline
\end{tabular}

Table 2. Association between infant MTR genetic polymorphisms and CHDs assuming various genetic models. *Odds ratios adjusted by maternal age, maternal education level, fetal gender. ${ }^{\$}$ Three models adjusted by multiple testing correction of false discovery rate. 18 polymorphisms in seven genes were covered by this correction.

\begin{tabular}{|l|l|l|l|l|l|l|}
\hline Marker & Subtype & Transmitted & Not transmitted & OR(95\%CI) & $\chi^{\mathbf{2}}$ & $\boldsymbol{P}_{m}{ }^{*}$ \\
\hline \multirow{4}{*}{ rs1770449 } & Total & 73 & 32 & $2.281(1.506,3.457)$ & 16.010 & $\mathbf{0 . 0 0 1}$ \\
\cline { 2 - 7 } & SPD & 58 & 25 & $2.320(1.452,3.708)$ & 13.120 & $\mathbf{0 . 0 0 7}$ \\
\cline { 2 - 7 } & CTD & 44 & 11 & $4.000(2.066,7.745)$ & 19.800 & $<\mathbf{0 . 0 0 1}$ \\
\hline \multirow{3}{*}{ rs1050993 } & Total & 73 & 32 & $2.281(1.506,3.457)$ & 16.010 & $\mathbf{0 . 0 0 1}$ \\
\cline { 2 - 7 } & SPD & 58 & 25 & $2.320(1.452,3.708)$ & 13.120 & $\mathbf{0 . 0 0 7}$ \\
\cline { 2 - 7 } & CTD & 44 & 11 & $4.000(2.066,7.745)$ & 19.800 & $<\mathbf{0 . 0 0 1}$ \\
\hline
\end{tabular}

Table 3. Transmission of MTR variant alleles from heterozygous case-parent triads. ${ }^{\sharp} P_{\mathrm{m}}: P$ value adjusted by permutation test.

\begin{tabular}{|l|l|l|l|l|l|}
\hline Haplotype & Subtype & Transmitted & Not transmitted & $\chi^{\mathbf{2}}$ & P \\
\hline \multirow{4}{*}{ MTR 3-SNP: rs1770449-rs1805087-rs1050993 } & \multicolumn{4}{|l|}{} \\
\hline \multirow{4}{*}{ CAA } & Total & 73 & 32 & 16.010 & $<\mathbf{0 . 0 0 1}$ \\
\cline { 2 - 6 } & SPD & 58 & 25 & 13.120 & $<\mathbf{0 . 0 0 1}$ \\
\cline { 2 - 6 } & CTD & 44 & 11 & 19.800 & $<\mathbf{0 . 0 0 1}$ \\
\hline \multirow{4}{*}{ TGG } & Total & 24 & 31 & 0.891 & 0.345 \\
\cline { 2 - 7 } & SPD & 17 & 23 & 0.900 & 0.343 \\
\cline { 2 - 6 } & CTD & 12 & 15 & 0.333 & 0.564 \\
\hline \multirow{4}{*}{ TAG } & Total & 53 & 87 & 8.257 & $\mathbf{0 . 0 0 4}$ \\
\cline { 2 - 6 } & SPD & 40 & 53 & 6.813 & $\mathbf{0 . 0 0 9}$ \\
\cline { 2 - 6 } & CTD & 23 & 11.840 & $\mathbf{0 . 0 0 1}$ \\
\hline
\end{tabular}

Table 4. Transmission of MTR haplotype from heterozygous case-parent triads.

1.656-6.429) or 3.263(95\%CI: 1.656-6.429) times greater than the aOR in women carrying wild genotype, respectively.

Further analysis found additive-scale interactions between the above mentioned SNPs (rs1770449: $\mathrm{S}=0.066$, RERI $=-2.227$; rs1050993: $\mathrm{S}=0.078, \mathrm{RERI}=-2.186$ ) and maternal folate intake (Table 5).

\section{Discussion}

In this family-based association study, over-transmission of SNPs rs1770449 and rs1050993 and haplotype CAA (rs1770449-rs1805087-rs1050993) in MTR were detected in total CHDs, SPD and CTD groups. Significant interactions between maternal periconceptional folic acid supplementation and foetal SNPs rs1770449 and rs1050993 on the risk of CHDs were observed. 


\begin{tabular}{|c|c|c|c|c|c|c|c|}
\hline Marker & Genotype & $\begin{array}{l}\text { Maternal } \\
\text { folate intake }\end{array}$ & Case & Control & $\mathrm{aOR}^{\mathrm{a}}(95 \% \mathrm{CI})$ & $P_{1}{ }^{\mathrm{b}}$ & $P_{2}^{c}$ \\
\hline \multirow{4}{*}{ rs1770449 } & TT & no & 40 & 60 & Ref. & 0.015 & 0.014 \\
\hline & \begin{tabular}{|l|} 
TT \\
\end{tabular} & yes & 42 & 81 & $1.123(0.619,2.039)$ & & \\
\hline & CT or $\mathrm{CC}$ & no & 51 & 26 & $3.262(1.656,6.429)$ & & \\
\hline & CT or CC & yes & 27 & 41 & $1.158(0.585,2.295)$ & & \\
\hline \multirow{4}{*}{ rs1050993 } & GG & no & 40 & 60 & Ref. & 0.019 & 0.016 \\
\hline & GG & yes & 42 & 82 & $1.109(0.611,2.012)$ & & \\
\hline & AG or AA & no & 51 & 26 & $3.263(1.656,6.429)$ & & \\
\hline & AG or AA & yes & 27 & 40 & $1.185(0.597,2.354)$ & & \\
\hline
\end{tabular}

Table 5. Interaction between maternal folic acid supplement and infant genotypes of MTR gene on the risk of CHDs. ${ }^{a}$ Odds ratios adjusted for maternal age, maternal education level, fetal gender. ${ }^{b} \mathrm{P}_{1}$ : counted by logistic regression. ${ }^{c} \mathrm{P}_{2}$ : counted by cross analysis.

Our results indicated that MTR may be associated with the risk of CHDs, which was identified in both case-control and TDT analyses. To our knowledge, these SNPs have not been previously reported in association with CHDs. In a study with $2340 \mathrm{CHD}$ cases and 2270 controls performed in China, researchers found that two regulatory variants of $M T R,-186 \mathrm{~T}>\mathrm{G}$ and $+905 \mathrm{G}>\mathrm{A}$, were associated with an increased risk of $\mathrm{CHD}$. Both of the minor alleles were correlated with elevated plasma homocysteine concentrations, indicating a genetic component for hyperhomocysteinaemia ${ }^{11}$. Previous studies indicated that CHD is related to a high homocysteine (Hcy) level ${ }^{14}$ or hyperhomocysteinaemia, which leads to a cardiovascular damage in the early development period ${ }^{15,16}$. MTR is a key enzyme in folate metabolism that catalyses the transfer of a methyl group from 5-methyltetrahydrofolate to Hcy and removes Hcy to methionine ${ }^{7}$. A study in the Thai population found an increased risk of breast cancer for homozygotes in the MTR SNPs (rs1770449 and rs1050993) with the OR=2.21 and $\mathrm{OR}=2.24$, respectively ${ }^{17}$. Moreover, homozygous $M T R$ knock-out mice are embryonically lethal, suggesting that MTR activity is essential for early embryonic development ${ }^{18}$.

In addition, the activation of MTR requires for MTRR gene and it has been demonstrated that the MTRR c. $56+781 \mathrm{~A}>\mathrm{C}$ variant results in functionally reduced $M T R R$ expression at the transcriptional level ${ }^{12}$. Furthermore, MTRR regenerates the functional status of $M T R$ via the chemical reduction of vitamin $\mathrm{B} 12^{19}$. Therefore, both CUBN, which encodes the intrinsic factor-vitamin B12 receptor, and TCNI, a vitamin B12 binding protein, participate in the remethylation of $\mathrm{Hcy}^{20,21}$. In this study, our finding did not support a role of $M T R R$, CUBN and other included folate pathway genes in modifying the risk of CHDs in Chinese women, implying that studies with larger sample sizes are needed.

In our research, we further analysed the interaction of folate metabolism pathway genes and maternal folate intake on the risk of CHD and found that two SNPs (rs1770449 and rs1050993) in infants can modify the association between maternal folic acid intake and CHD risk. Folate is a kind of B vitamin and one-carbon donor, that plays an important role in DNA synthesis and cell division ${ }^{22}$. Nonetheless, folate cannot be synthesized in the body. Our study indicated that periconceptional folic acid supplementation was associated with a decreased risk of CHD, which is consistent with other studies ${ }^{23-25}$. Furthermore, our results suggested that this association could be modified by variants of the infant MTR gene. Hobbs reported that obese women carrying the TT genotype of MTHFR were 4.6 times more likely to have an affected pregnancy compared with normal-weight women carrying a CC genotype ${ }^{4}$. Women who smoked, were in the highest quartile of homocysteine concentration and had the MTHFR CC genotype, had a 12 -fold increased CHD risk compared with the reference group ${ }^{26}$. Other studies found an interaction between RFC1 80GG and folic acid intake on the risk of neural tube defects ${ }^{27}$.

There are some major strengths in this study. First, we performed a combination of case-control and case-parental control design, which allows both case-control association and TDT analysis of the cases. TDT, a generally recommended method, can effectively avoid the bias caused by population stratification ${ }^{28,29}$. The combination of the two study designs is cost-effective and robust, especially when the case-control and TDT results coincide with each other, offering greater confidence in the result ${ }^{30}$. Second, both multivariate logistic regression analysis and cross analysis were used to analyze the interaction between maternal folate intake and SNPs on the risk of CHDs. Researchers argued that interaction estimated as departure from additivity could better reflect a biologic interaction ${ }^{31,32}$. Third, the epidemiological data were collected in the first trimester to avoid recall bias. Fourth, the CHD cases were validated by following up one year after delivery. The limitations include that the study was performed in a single hospital, and the data about maternal environmental exposure were collected by self-reporting, not by the biomarker-based approach.

In summary, our study indicated that MTR SNPs rs 1770449 and rs1050993 and their haplotypes may be associated with the risk of CHDs. To the best of our knowledge, no other study has reported the association between these SNPs and the risk of CHDs. Moreover, rs1770449 and rs1050993 could also modify the association between maternal folic acid intake and CHD risk, which indicated that mothers with foetuses carrying the $\mathrm{C}$ allele of rs1770449 and the A allele rs1050993 should take folic acid per their doctor's recommendation. Our findings provide new evidence about $\mathrm{CHD}$ pathogenesis and enrich the knowledge of the impact of folate pathway genetic variants on CHDs, which are of great value for CHD clinical intervention. The results need to be verified in further large-scale studies and functional studies. 


\section{Materials and Methods}

Study population and data collection. The study was performed at the West China Second University Hospital from January 2014 to September 2015. A total of 160 CHD case-parent triads and 208 control-parent triads were recruited. Mothers with a singleton pregnancy diagnosed with CHD by echocardiography without extracardiac abnormalities, syndromic diseases and chromosomal aberrations were selected in the case group. Stillbirths were aborted according to standard process, and livebirths were followed up one year after delivery. Foetuses or infants with cardiomyopathy, cardiac rhabdomyoma, single umbilical artery or arrhythmia were excluded from the study. Mothers with a singleton pregnancy without any congenital malformations were recruited to the control group and matched by residence area and ethnicity.

All cases were classified into three subgroups based on the origin of heart development as follows: (1) septal defects (SPD); (2) conotruncal heart defects (CTD), including conotruncal, right-sided obstructive, left-sided obstructive heart defects and (3) other cardiac abnormalities. When performing subgroup analyses, we focused on SPD and CTD groups, because the sample size of other cardiac abnormalities was too small to count.

All the participants accepted a face-to-face interview in the first, second and third trimesters and 30 days after delivery. The questionnaires were composed of parental epidemiological characteristics, maternal diet and nutrition, living and working environment, etc. Maternal folate intake means mothers taking folic acid over 90 days or more during the 3 months before pregnancy to the first trimester ${ }^{33}$.

This research was approved by the Ethics Committee of West China Second University Hospital and was based on the tenets of the Declaration of Helsinki. Informed consent was collected from all participants.

Marker selection and genotyping. Eighteen SNPs in seven folate pathway genes, including MTR, MTRR, $B H M T, B H M T 2, C U B N, T C N 1$, and TCN2 were selected based on (1) minor allele frequency $(>0.05)$ and (2) association with CHDs or other congenital abnormalities in past studies ${ }^{7,11,21,34}$. Genomic DNA was extracted from peripheral blood according to the manufacturer (QIAamp DNA Blood Mini Kit), and SNPs were genotyped using a commercial custom-by-design $2 \times 48$-Plex SNPscan ${ }^{\mathrm{TM}}$ Kit (Cat\#: G0104, Genesky Biotechnologies Inc., Shanghai, China), as described previously ${ }^{35}$. Genotyping was performed in a single-blind approach and the sample grouping was unknown to laboratory staff. Ten percent of samples were randomly repeated for genotyping.

Statistical analysis. The demographic characteristics of the different groups were compared by chi-squared test using Statistical Package for Social Sciences (SPSS) version 21.0 software (SPSS Inc., IBM, Chicago, USA). A chi-squared test was performed to investigate the associations between allele frequencies and CHDs, and unconditional logistic regression analysis was used to investigate the associations between genotypes and CHDs assuming various genetic models (dominant, recessive and additive), respectively, in case and control groups using Plink software (http://pngu.mgh.harvard.edu/ purcell/plink/) in accordance with the Hardy-Weinberg equilibrium in the controls. False discovery rate (FDR) correction of multiple hypothesis testing was performed ${ }^{36}$.

Interactions between maternal folate supplement and infant genotypes on the risk of CHDs were assessed by multiple logistic regression analysis and cross analysis and adjusted by maternal age, maternal education level and foetal gender. Regression coefficient (B), value of Wald test (Wals), synergy index (S) and relative excess risk of interaction (RERI) were counted.

Transmission disequilibrium test(TDT) of alleles and haplotypes in 160 complete case-parent triads were analysed by Plink software. TDT was adjusted by permutation test and 10,000 permutations were conducted. The linkage disequilibrium (LD) patterns and haplotype structures were estimated using Haploview 4.2 software $^{37}$. Two-sided $P<0.05$ was considered statistically significant.

\section{References}

1. van der Linde, D. et al. Birth prevalence of congenital heart disease worldwide: a systematic review and meta-analysis. Journal of the American College of Cardiology 58, 2241-2247, https://doi.org/10.1016/j.jacc.2011.08.025 (2011).

2. Zhao, Q. M. et al. Prevalence of Congenital Heart Disease at Live Birth in China. The Journal of pediatrics 204, 53-58, https://doi. org/10.1016/j.jpeds.2018.08.040 (2019).

3. National Health and Family Planning Commission of PRC National Stocktaking Report on Birth defect Prevention. (2012).

4. Hobbs, C. A., Cleves, M. A., Karim, M. A., Zhao, W. \& MacLeod, S. L. Maternal folate-related gene environment interactions and congenital heart defects. Obstetrics and gynecology 116, 316-322, https://doi.org/10.1097/AOG.0b013e3181e80979 (2010).

5. Vecoli, C., Pulignani, S., Foffa, I. \& Andreassi, M. G. Congenital heart disease: the crossroads of genetics, epigenetics and environment. Current genomics 15, 390-399, https://doi.org/10.2174/1389202915666140716175634 (2014).

6. Shi, H. et al. Study on Environmental Causes and SNPs of MTHFR, MS and CBS Genes Related to Congenital Heart Disease. PloS one 10, e0128646, https://doi.org/10.1371/journal.pone.0128646 (2015).

7. Cai, B. et al. Genetic variant in MTRR, but not MTR, is associated with risk of congenital heart disease: an integrated meta-analysis. PloS one 9, e89609, https://doi.org/10.1371/journal.pone.0089609 (2014).

8. Botto, L. D., Olney, R. S. \& Erickson, J. D. Vitamin supplements and the risk for congenital anomalies other than neural tube defects. American journal of medical genetics. Part C, Seminars in medical genetics 125C, 12-21, https://doi.org/10.1002/ajmg.c.30004 (2004).

9. Huhta, J. C., Linask, K. \& Bailey, L. Recent advances in the prevention of congenital heart disease. Current opinion in pediatrics 18, 484-489, https://doi.org/10.1097/01.mop.0000245347.45336.d7 (2006).

10. Jenkins, K. J. et al. Noninherited risk factors and congenital cardiovascular defects: current knowledge: a scientific statement from the American Heart Association Council on Cardiovascular Disease in the Young: endorsed by the American Academy of Pediatrics. Circulation 115, 2995-3014, https://doi.org/10.1161/CIRCULATIONAHA.106.183216 (2007).

11. Zhao, J. Y. et al. Genetic variants reducing MTR gene expression increase the risk of congenital heart disease in Han Chinese populations. EurHeart J 35, 733-742, https://doi.org/10.1093/eurheartj/eht221 (2014).

12. Zhao, J. Y. et al. Functional variant in methionine synthase reductase intron-1 significantly increases the risk of congenital heart disease in the Han Chinese population. Circulation 125, 482-490, https://doi.org/10.1161/CIRCULATIONAHA.111.050245 (2012).

13. Pereira, A. C. et al. Lack of evidence of association between MTHFR C677T polymorphism and congenital heart disease in a TDT study design. International journal of cardiology 105, 15-18, https://doi.org/10.1016/j.ijcard.2004.10.049 (2005).

14. Hobbs, C. A. et al. Maternal homocysteine and congenital heart defects. Journal of the American College of Cardiology 47, 683-685, https://doi.org/10.1016/j.jacc.2005.11.013 (2006). 
15. Brosnan, J. T. Homocysteine and cardiovascular disease: interactions between nutrition, genetics and lifestyle. Canadian journal of applied physiology=Revue canadienne de physiologie appliquee 29, 773-780 (2004).

16. Huhta, J. C. \& Hernandez-Robles, J. A. Homocysteine, folate, and congenital heart defects. Fetal and pediatric pathology 24, 71-79, https://doi.org/10.1080/15227950591008240 (2005).

17. Sangrajrang, S. et al. Genetic polymorphisms in folate and alcohol metabolism and breast cancer risk: a case-control study in Thai women. Breast cancer research and treatment 123, 885-893, https://doi.org/10.1007/s10549-010-0804-4 (2010).

18. Swanson, D. A. et al. Targeted disruption of the methionine synthase gene in mice. Molecular and cellular biology 21, 1058-1065, https://doi.org/10.1128/MCB.21.4.1058-1065.2001 (2001).

19. Verkleij-Hagoort, A. C. et al. Genetic and lifestyle factors related to the periconception vitamin B12 status and congenital heart defects: a Dutch case-control study. Molecular genetics and metabolism 94, 112-119, https://doi.org/10.1016/j.ymgme.2007.12.002 (2008).

20. Johnston, J., Yang-Feng, T. \& Berliner, N. Genomic structure and mapping of the chromosomal gene for transcobalamin I (TCN1): comparison to human intrinsic factor. Genomics 12, 459-464 (1992).

21. Aminoff, M. et al. Mutations in CUBN, encoding the intrinsic factor-vitamin B12 receptor, cubilin, cause hereditary megaloblastic anaemia 1. Nature genetics 21, 309-313, https://doi.org/10.1038/6831 (1999).

22. Botto, L. Do multivitamin supplements reduce the risk for congenital heart defects? Evidence and gaps. Images in paediatric cardiology 2, 19-27 (2000).

23. Czeizel, A. E., Vereczkey, A. \& Szabo, I. Folic acid in pregnant women associated with reduced prevalence of severe congenital heart defects in their children: a national population-based case-control study. European journal of obstetrics, gynecology, and reproductive biology 193, 34-39, https://doi.org/10.1016/j.ejogrb.2015.06.024 (2015).

24. Liu, S. et al. Effect of Folic Acid Food Fortification in Canada on Congenital Heart Disease Subtypes. Circulation 134, 647-655, https://doi.org/10.1161/CIRCULATIONAHA.116.022126 (2016).

25. Li, X. et al. The association between periconceptional folic acid supplementation and congenital heart defects: a case-control study in China. Preventive medicine 56, 385-389, https://doi.org/10.1016/j.ypmed.2013.02.019 (2013).

26. Hobbs, C. A. et al. Congenital heart defects, maternal homocysteine, smoking, and the $677 \mathrm{C}>\mathrm{T}$ polymorphism in the methylenetetrahydrofolate reductase gene: evaluating gene-environment interactions. Am J Obstet Gynecol 194, 218-224, https:// doi.org/10.1016/j.ajog.2005.06.016 (2006).

27. Pei, L. J. et al. Interaction between maternal periconceptional supplementation of folic acid and reduced folate carrier gene polymorphism of neural tube defects. Zhonghua yi xue yi chuan xue za zhi=Zhonghua yixue yichuanxue zazhi=Chinese journal of medical genetics 22, 284-287 (2005).

28. Cardon, L. R. \& Palmer, L. J. Population stratification and spurious allelic association. Lancet 361, 598-604, https://doi.org/10.1016/ S0140-6736(03)12520-2 (2003)

29. Hopper, J. L., Bishop, D. T. \& Easton, D. F. Population-based family studies in genetic epidemiology. Lancet 366, 1397-1406, https:// doi.org/10.1016/S0140-6736(05)67570-8 (2005).

30. Ackerman, H. et al. A comparison of case-control and family-based association methods: the example of sickle-cell and malaria. Annals of human genetics 69, 559-565, https://doi.org/10.1111/j.1529-8817.2005.00180.x (2005).

31. Knol, M. J., van der Tweel, I., Grobbee, D. E., Numans, M. E. \& Geerlings, M. I. Estimating interaction on an additive scale between continuous determinants in a logistic regression model. International journal of epidemiology 36, 1111-1118, https://doi. org/10.1093/ije/dym157 (2007).

32. Rothman, K. J., Greenland, S. \& Lash, T. L. Modern epidemiology [M] . Lippincott: Williams \& Wilkins (2008).

33. Shuyi Yuan, H. Y. \& Zeng, L. etc. Association between congenital heart disease and folic acid supplementation during periconceptional period among women of childbearing age in Shaanxi. Journal of Xian Jiaotong University(Medical Sciences) 38, 343-347 (2017)

34. Mostowska, A., Hozyasz, K. K., Biedziak, B., Misiak, J. \& Jagodzinski, P. P. Polymorphisms located in the region containing BHMT and BHMT2 genes as maternal protective factors for orofacial clefts. European journal of oral sciences 118, 325-332, https://doi. org/10.1111/j.1600-0722.2010.00757.x (2010).

35. Chen, X. et al. Genome-wide association study validation identifies novel loci for atherosclerotic cardiovascular disease. Journal of thrombosis and haemostasis: JTH 10, 1508-1514, https://doi.org/10.1111/j.1538-7836.2012.04815.x (2012).

36. Jiang, W. \& Yu, W. Jointly determining significance levels of primary and replication studies by controlling the false discovery rate in two-stage genome-wide association studies. Statistical methods in medical research 27, 2795-2808, https://doi. org/10.1177/0962280216687168 (2018).

37. Barrett, J. C. Haploview: Visualization and analysis of SNP genotype data. Cold Spring Harbor protocols 2009, pdb ip71, https://doi. org/10.1101/pdb.ip71 (2009).

\section{Acknowledgements}

We thank all the families participating in this research and are grateful to obstetricians, paediatricians, pathologists, experimental technicians and other participants involved in the project. This work was supported by Grant 81373069 from the National Natural Science Foundation of China.

\section{Author Contributions}

C.D. performed statistical work and drafted manuscript. L.D. and H.L. designed research plan and revised manuscript. Y.D. and L.X. prepared the blood samples. L.Y. and L.L. performed field investigation and followed up. All authors had read and approved the final manuscript.

\section{Additional Information}

Supplementary information accompanies this paper at https://doi.org/10.1038/s41598-019-41641-z.

Competing Interests: The authors declare no competing interests.

Publisher's note: Springer Nature remains neutral with regard to jurisdictional claims in published maps and institutional affiliations. 
(i) Open Access This article is licensed under a Creative Commons Attribution 4.0 International License, which permits use, sharing, adaptation, distribution and reproduction in any medium or format, as long as you give appropriate credit to the original author(s) and the source, provide a link to the Creative Commons license, and indicate if changes were made. The images or other third party material in this article are included in the article's Creative Commons license, unless indicated otherwise in a credit line to the material. If material is not included in the article's Creative Commons license and your intended use is not permitted by statutory regulation or exceeds the permitted use, you will need to obtain permission directly from the copyright holder. To view a copy of this license, visit http://creativecommons.org/licenses/by/4.0/.

(C) The Author(s) 2019 